\title{
Can Computational Design Replace Human Design of Antimicrobial Peptides?
}

\author{
Cheng JTJ* \\ Department of Microbiology and Immunology, University of British Columbia, \\ Canada
}

*Corresponding author: John Tien Jui Cheng, Department of Microbiology and Immunology, University of British Columbia, Canada, Email: jtj_cheng@yahoo.com

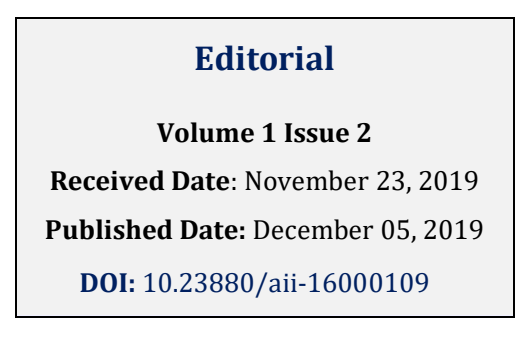

Abbreviations: AMP: Antimicrobial Peptides; APD: Antimicrobial Peptide Database; GRAMPA: Giant Repository Antimicrobial Peptides Activity; MIC: Minimum Inhibitory Concentration; CNN: Convolutional Neural Network.

\section{Editorial}

Resistance to small molecule antibiotics has posed a major threat to healthcare due to lack of available next generation antibiotics. Particularly, the infamous "superbugs" infections are not only difficult to treat, but also present high reoccurring rates and persistent biofilm problems, causing complication in treatment and prevention in community, hospital, as well as other setting.

Antimicrobial peptides (AMPs) are a class of naturally occurring peptides produced by organisms across multiple kingdoms to defend the host against the invading pathogen. AMPs offer great alternatives to develop the next generation antimicrobial agents. Today, there are 3140 naturally occurring and synthetic antimicrobial peptides identified from six kingdoms according to The Antimicrobial Peptide Database (APD) [1-3], presenting a good arsenal against microbial infection and providing a wide selection for antimicrobial drug development.

Computational approach to design new antimicrobial peptides can be dated to early 2000. Due to many shared biophysical properties and similar mechanisms of actions, computational design and machine learning have become very popular tools to generate new, ideally more active peptides [4,5]. To date, several methods have been developed for computational design of antimicrobial peptides. Generally, these can be categorized into two approaches: classification algorithms and regression approaches. Classification algorithms predict if a peptide will be antimicrobial or not based on the peptide sequence. Examples are AMP Scanner v2 and AMP pred [6,7]. Regression approaches predict quantitatively the antimicrobial activity of a candidate peptide based on peptide structure and biophysical properties, oftentimes used to optimize a local sequence and/or a specific known scaffold $[8,9]$.

While both approaches are valid and well-practiced, these are the questions that we should really ask ourselves: Can computer algorithms think independently without human input? Without human input, can computer algorithms think independently to provide objective recommendation?. With all advances in artificial intelligence, can a computer design an algorithm on its own without human intervention and manipulation?.

Other areas that are often neglected by the computational design strategy are prediction of toxicity, pharmacodynamics and pharmacokinetic profiles, therapeutic index optimization in the clinical setting (in comparison and complement with therapeutic index obtained in vitro), physico chemical properties hindering successful formulation and trade-off with antimicrobial activity. These problems are often detected by human experiments and observation. Recently, additional algorithms have been developed to address the prediction 


\section{Annals of Immunology \& Immunotherapy}

of in vitro toxicity; however these are still not sufficient to predict in vivo toxicity [10].

The most recent Giant Repository of AMP Activity (GRAMPA) algorithm by Witten and Witten at MIT was developed to improve the above-mentioned approaches by combining a large dataset with a regression model to design AMPs with a low predicted minimum inhibitory concentration (MIC), and then further train convolutional neural network (CNN) models for AMP activity prediction [11]. While this approach yields promising candidates with novel sequences, the same prediction problems will still need to be resolved prior to preclinical studies. Until these problems can be resolved, we still have a long journey toward effective computational design of antimicrobial peptides without a need for experimental validation.

The general consensus in antimicrobial research mostly perceives computational approaches as a next generation tool to objectively design novel antimicrobial peptides. In reality, how objective machine learning and artificial intelligence can be and will be still remains undetermined. Moreover, machine learning and artificial intelligence lack human creativity, and ironically this creativity is often needed in scientific research, albeit contrary to much desired objectivity in the scientific research community. We need to move beyond designing more active antimicrobial peptides via classification algorithms and regression approaches by iteratively improving our prediction algorithms of other preclinical studies parameters in complement. Using computational design is a good strategy to find novel antimicrobials to overcome antibiotic resistance problems among many others but is still far away from replacing human design. In my opinion, both computational and human designs are complementary and mutually inclusive. We need both approaches to identify next generation antimicrobials to continue this ongoing battle.

\section{References}

1. Wang, Z, Wang G (2004) APD: the Antimicrobial Peptide Database. Nucleic Acids Res 32(1): 590-592.
2. Wang G, Li X, Wang Z (2009) APD2: the updated antimicrobial peptide database and its application in peptide design. Nucleic Acids Res 37: 933-937.

3. Wang G, Li X, Wang Z (2016) APD3: the antimicrobial peptide database as a tool for research and education. Nucleic Acids Res 44(1): 1087-1093.

4. Nguyen LT, Haney EF, Vogel HJ (2011) The expanding scope of antimicrobial peptide structures and their modes of action. Trends Biotechnol 29(9): 464-472.

5. Lee TH, Hall KN, Aguilar MI (2016) Antimicrobial Peptide Structure and Mechanism of Action: A Focus on the Role of Membrane Structure. Curr Top Med Chem 16(1): 25-39.

6. Veltri D, Kamath U, Shehu A (2018) Deep learning improves antimicrobial peptide recognition. Bioinformatics 34(16): 2740-2747.

7. Meher PK, Sahu TK, Saini V, Rao AR (2017) Predicting antimicrobial peptides with improved accuracy by incorporating the compositional, physico-chemical and structural features into Chou's general PseAAC. Sci Rep 7: 42362.

8. Yoshida M, Hinkley T, Tsuda S, Abul Haija YM, McBurney RT, et al. (2018) Using Evolutionary Algorithms and Machine Learning to Explore Sequence Space for the Discovery of Antimicrobial Peptides. Chem 4(3): 533-543.

9. Hilpert K, Elliott MR, Volkmer Engert R, Henklein P, Donini O, et al. (2006) Sequence requirements and an optimization strategy for short antimicrobial peptides. Chem Biol 13(10): 1101-1107.

10. Gupta S, Kapoor P, Chaudhary K, Gautam A, Kumar R, et al. (2015) Peptide toxicity prediction. Methods Mol Biol 1268: 143-157.

11. Witten J, Witten Z (2019) Deep learning regression model for antimicrobial peptide design. CSH bioRxiv. 